\title{
Elemente der Planeten (299) und (300).
}

Planet (299)

entdeckt in Wien von Hrn. Dr. J. Palisa am 6. Oct. I890.

Zur Berechnung der Elemente sind 4 Wiener Beobachtungen benutzt worden, und zwar Oct. 6, Oct. 2 I, Nov. 4 und Dec. 8. Herr Dr. Palisa hat den Planeten dann noch bis Anfang Januar I891 verfolgen können.

Epoche 1890 Dec. 8.5 M. Z. Berlin

$$
\begin{aligned}
& M=\text { I I }^{\circ} 15^{\prime} 55^{\prime \prime} 3 \\
& \omega=\begin{array}{lll}
15 \mathrm{I} & 2 & 2.8
\end{array} \\
& \delta=24 I \quad 42 \quad 54.4 \quad \text { M. Aequ. } 1890.0 \\
& i=135 \text { 10.1 } \\
& \varphi=33 \text { I } 34.5 \\
& \mu=935^{\circ .234} \\
& \log a=0.38605^{8}
\end{aligned}
$$

Planet (300)

zuerst von Herrn A. Charlois in Nizza am 3. Oct. I 890 beobachtet und für $(298)$ gehalten, konnte ebenfalls noch bis

\begin{tabular}{|c|c|c|c|c|c|c|}
\hline \multirow{2}{*}{1890} & \multicolumn{2}{|c|}{$(298)$} & \multicolumn{2}{|c|}{$(300)$} & \multicolumn{2}{|c|}{$(3 \circ 2)$} \\
\hline & $\alpha$ & $\delta$ & $\alpha$ & $d$ & $\alpha$ & $\delta$ \\
\hline Sept. $\quad 9.5$ & $0^{b} 3^{8^{m}} 3^{s}$ & $+2^{\circ} 41: 4$ & $0^{h} 34^{m} 39^{s}$ & $+2^{\circ} 56: 7$ & $o^{h} 34^{m} 53^{s}$ & $+2^{\circ} 49^{\prime} 6$ \\
\hline 13.5 & 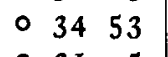 & +229.7 & $\circ 328$ & +240.9 & $\begin{array}{lll}0 & 31 & 49\end{array}$ & $+237 \cdot 3$ \\
\hline 17.5 & $\begin{array}{lll}0 & 3^{x} & 5\end{array}$ & +216.6 & $\begin{array}{lll}0 & 2927\end{array}$ & $+224 \cdot I$ & $\begin{array}{lll}0 & 28 & 26\end{array}$ & +223.5 \\
\hline
\end{tabular}
in den Januar 189 r beobachtet werden. Die Elemente beruhen auf vier Oertern, Oct. 3 Nizza, Oct. 22 und Nov. I Wien; endlich Nov. I 7 Nizza.

$$
\begin{aligned}
& \text { Epoche } 1890 \text { Nov. } 17.5 \text { M. Z. Berlin. } \\
& M=3^{8 \circ} 26^{\prime} \text { × } 3 \text { "०。 } \\
& \omega=290 \quad 4^{8} \quad 36.5 \\
& \delta=\begin{array}{llll}
42 & 27 & 19.8 & \text { M. Aequ. } 1890.0
\end{array} \\
& i=04644 . \mathrm{I} \\
& \varphi=2233^{8.7} \\
& \mu=617.2617 \\
& \log a=0.5063582
\end{aligned}
$$

Es ist merkwürdig, wie nahe die drei Planeten (298), (300) und (302) einander im September 1890 gestanden haben, ohne dass die zwei letzteren entdeckt wurden. Die bisherigen Elemente geben folgende Oerter:

Berlin $189 \mathrm{I}$ Febr. 23.

\section{A. Berberich}

\section{Wolsingham Observatory Circular No. 30 .}

The variability of Es. Birm. $146=$ DM. $+68: 398$ has been ascertained. Period and limit unknown. T. E. Espin.

Beobachtung des Planeten (306), entdeckt von Charlois Febr. 16.

Telegramm vom 27 . Februar: I 89 I Febr. $268^{\mathrm{h}} 19^{\mathrm{m}} \cdot 2$ M. Z. Hamburg AR. app. $=143^{\circ} 35^{\prime}$ 1 $3^{\prime \prime} ;$ PD. app. $=82^{\circ} 6^{\prime} 47^{\prime \prime}$ W. Luther.

\section{Anzeige.}

Die Herren Abonnenten, welche die Astronomischen. Nachrichten ferner zu erhalten wünschen, werden ersucht ihre Bestellung und Vorausbezahlung auf den folgenden Band baldmöglichst einzusenden, wofern es der Expedition nicht bekannt ist, dass sie als ständige Abonnenten angesehen werden wollen.

Man pränumerirt bei der Expedition der Astronomischen Nachrichten, Sternwarte Kiel, mit netto I 2 Mark für den Band von 24 Nummern nebst Inhaltsverzeichniss und Register. Für die von der Expedition nummerweise franco versandten Exemplare beträgt der Preis 15 Mark. Einzelne Nummern werden zur Completirung, wenn sie vorräthig sind, zum Preise von 6o Pfennig abgelassen.

Den Hauptdebit dieses Blattes hat, wie bisher, die Buchhandlung von W. Mauke Söhne in Hamburg.

Geschlossene Bände, von Band roo an, können jederzeit von der Expedition, Sternwarte Kiel, zum Preise von 12 Mark pro Band bezogen werden. Von den älteren Bänden sind noch Band 32-99 vorräthig und zu gleichem Preise bei Prof. C. F. W. Peters, Königsberg in Pr., verkäuflich.

Inhalt zu Nr. 3022. C. Mönnichmeyer. Allgemeine Störungen der Themis durch Mars und Saturn. 345. - F. H. Franke. Ueber die Transformation rechtwinklig - sphärischer (geodätischer) Coordinaten auf neue Normalpunkte. $353 .-7$. Tebbutt. Observations of Comets at Windsor N, S. Wales, 357. - A. Berberich. Elemente der Planeten (299) und (300). 359. - T.E. Espin. Wolsingham Circular No. 30. 359. - W. Luther. Beobachtung des Planeten (306). 359. - Anzeige. 359. 